\title{
molecules
}

ISSN 1420-3049

www.mdpi.com/journal/molecules

Article

\section{In Vitro Synergy of Biochanin A and Ciprofloxacin against Clinical Isolates of Staphylococcus aureus}

\section{Guoxing Liu ${ }^{1, \dagger}$, Jun-Chao Liang ${ }^{1, \dagger}$, Xue-Lin Wang ${ }^{1, \dagger}$, Zhao-Hui Li ${ }^{1, \dagger}$, Wei Wang ${ }^{1, \dagger}$, Na Guo ${ }^{2, \dagger}$, Xiu-Ping Wu ${ }^{1, \dagger}$, Feng-Ge Shen ${ }^{1}$, Ming-Xun Xing ${ }^{1}$, Li-Hui Liu ${ }^{1}$, Lei Li ${ }^{1}$, Ming-Yuan Liu ${ }^{1}$ * and $L u Y u^{1,3,4, *}$}

1 Key Laboratory of Zoonosis Research, Ministry of Education, Institute of Zoonosis, College of Animal Science and Veterinary Medicine, Jilin University, Changchun 130062, China

2 Department of Food Quality and Safety, College of Quartermaster Technology, Jilin University, Changchun 130062, China

3 Institute of Pathogen Biology, Chinese Academy of Medical Sciences, Beijing 100730, China

4 State Key Laboratory for Molecular Virology and Genetic Engineering, Chinese Center for Disease Control and Prevention, Beijing 100176, China

$\dagger$ These authors contributed equally to this work.

* Authors to whom correspondence should be addressed; E-Mails: liumy36@163.com (M.L.); yulu225@yahoo.com.cn (L.Y.); Tel.: +86-431-87836702 (M.L.); +86-431-87836713 (L.Y.); Fax: +86-431-87836702 (M.L.); +86-431-87836160 (L.Y.).

Received: 11 July 2011; in revised form: 30 July 2011 / Accepted: 2 August 2011 /

Published: 5 August 2011

Abstract: Many clinical isolates of Staphylococcus aureus (S. aureus) are resistant to numerous antimicrobials, including the fluoroquinolones (FQs). Flavonoids such as biochanin A (BCA) are compounds that are naturally present in fruits, vegetables, and plant-derived beverages. The goal of this investigation was to study the possible synergy between the antimicrobial agents BCA and ciprofloxacin (CPFX) when used in combination; CPFX was chosen as a representative FQ compound. We used $S$. aureus strain ATCC 25923 and 11 fluoroquinolone (FQ)-resistant methicillin-resistant S. aureus (MRSA) strains. Results from the drug susceptibility testing and checkerboard assays show that the minimum inhibitory concentration (MIC) of BCA ranged from $64 \mu \mathrm{g} / \mathrm{mL}$ to $512 \mu \mathrm{g} / \mathrm{mL}$. When BCA was combined with CPFX, the fractional inhibitory concentration index (FICI) data showed that there was synergy in all 12 of the S. aureus strains tested. No antagonistic activity was observed in any of the strains tested. The results of time-kill 
tests and agar diffusion tests confirm that there was synergy between BCA and CPFX against $S$. aureus strains. These results suggest that BCA can be combined with FQs to produce a powerful antimicrobial agent.

Keywords: antimycobacterial; biochanin A; ciprofloxacin; Staphylococcus aureus; synergy

\section{Introduction}

Staphylococcus aureus (S. aureus) is a significant community and nosocomially acquired pathogen that colonizes the nasal passages and skin of healthy people [1]. S. aureus colonization can lead to local and systemic infections in humans, which can range from superficial skin infections to life-threatening pneumonia or sepsis [2]. The widespread use of methicillin and other semi-synthetic penicillins in the late 1960s led to the emergence of the strains of methicillin resistant S. aureus (MRSA) [3]. Current more than $60 \%$ of $S$. aureus isolates are resistant to methicillin, and a number of strains have developed resistance to more than 20 other antimicrobial drugs [4], including the fluoroquinolones (FQs). Because of this rapidly evolving drug resistance, the morbidity and mortality associated with S. aureus infections is high in spite of antimicrobial therapy [5]. A novel approach for treating resistant strains of bacteria is to use a combination therapy, which may improve the efficacy of antimicrobial treatment for resistant microbes.

Antimicrobials can have detrimental side effects, and as mentioned previously, their overuse can lead to bacterial resistance. Thus, plant-derived products have been explored for use as alternative treatments [6,7]. In vitro studies have shown that several flavonoids have a strong antimicrobial activity against gram-positive bacteria [6,8]. Biochanin A (BCA, Figure 1), an isoflavonoid found in chickpea (Cicer arietinum), in peanuts and in other legumes, inhibits the growth of various microorganisms [9-12]. BCA is classified as a phytoestrogen and has putative benefits in dietary cancer prophylaxis [13]. It acts as a chemopreventive agent by inhibiting the metabolism of benzo(a)pyren, which is the initial step in its carcinogenicity [14-16]. In addition, BCA was found to be potent agonists of aryl hydrocarbon receptor in vitro, which activation affects cell cycle control and apoptosis [13]. As a common plant product, BCA is generally considered to be nontoxic [12]. Our preliminary data show that BCA has antimicrobial activity against several $S$. aureus strains. These interesting results motivated us to further study possible synergy between BCA and a representative FQ (CPFX) against several drug resistant $S$. aureus isolates. In this study, we investigated the combined effects of BCA and CPFX against clinical isolates of $S$. aureus with the checkerboard microdilution method, time-killing tests, and agar diffusion tests.

Figure 1. Chemical formula of Biochanin A.<smiles>COc1ccc(-c2coc3cc(O)cc(O)c3c2=O)cc1</smiles> 


\section{Results}

\subsection{Drug Effect Alone and in Combination}

In the assessment of the antimicrobial activities of the drug alone, the MIC values for BCA treatment alone against the 11 clinical isolates ranged from 128 to $512 \mu \mathrm{g} / \mathrm{mL}$; the MIC values for CPFX treatment alone against the 11 clinical isolate strains ranged from 128 to $256 \mu \mathrm{g} / \mathrm{mL}$ (Table 1), and these large values show that the clinical MRSA isolates are resistant to CPFX and possibly a majority of the FQs. The MIC values of BCA and CPFX against ATCC 25923 strain were 64 and $1 \mu \mathrm{g} / \mathrm{mL}$, respectively, and the CPFX MIC value is within the reference range. These results show that BCA by itself has weak antimicrobial activity against the drug-resistant clinical $S$. aureus isolates.

Table 1. Susceptibilities of BCA alone and in combination with CPFX against $S$. aureus strains, as obtained by the spectrophotometric method.

\begin{tabular}{ccccc}
\hline \multirow{2}{*}{ Strains } & \multicolumn{4}{c}{ Median MIC (range) of drug $(\boldsymbol{\mu g} / \mathbf{m L} \mathbf{c}$} \\
\cline { 2 - 5 } & \multicolumn{2}{c}{ Alone } & \multicolumn{2}{c}{ In combination } \\
\cline { 2 - 5 } & $256(128-256)$ & $256(256)$ & $32(32-64)$ & $32(32-64)$ \\
\hline S. aureus 1662 & $128(128-256)$ & $256(256)$ & $16(8-16)$ & $8(8)$ \\
S. aureus 3633 & $128(128)$ & $256(128-256)$ & $32(16-32)$ & $64(32-64)$ \\
S. aureus 3814 & $256(256-512)$ & $256(256)$ & $32(32-64)$ & $16(16-32)$ \\
S. aureus 3725 & $128(128-256)$ & $256(128-256)$ & $8(8-16)$ & $16(8-16)$ \\
S. aureus 2855 & $128(128-256)$ & $256(256)$ & $32(32-64)$ & $32(32-64)$ \\
S. aureus 1881 & $256(256)$ & $256(128-256)$ & $32(32-64)$ & $32(32-64)$ \\
S. aureus 3014 & $256(128-256)$ & $128(128-256)$ & $64(32-64)$ & $32(32-64)$ \\
S. aureus 3115 & $256(256-512)$ & $256(256-512)$ & $32(32-64)$ & $8(8-16)$ \\
S. aureus 3451 & $256(256-512)$ & $256(256)$ & $64(64-128)$ & $32(32)$ \\
S. aureus 3182 & $512(256-512)$ & $256(128-256)$ & $64(32-64)$ & $32(32-64)$ \\
S. aureus 1787 & $64(64)$ & $1(1)$ & $16(4-16)$ & $0.25(0.25)$ \\
S.aureus ATCC 25923 & & & &
\end{tabular}

In the combination study, after the background $\mathrm{OD}_{660}$ obtained from the microorganism-free well had been subtracted, the percentages of bacterial growth for the combination were calculated by comparing the $\mathrm{OD}_{660}$ of the drug-containing well with that of the drug-free well. In the checkerboard assay, FICI was calculated to analyze the interaction between the two drugs (BCA and CPFX) when combined. Of the 12 total $S$. aureus strains that were tested, the interaction between BCA and CPFX was synergistic in all of the strains according to the FICI method (all of the FICI values ranged from 0.06 to 0.5 ) (Table 2). We did not find any antagonistic interaction between BCA and CPFX.

From the data above, we conclude that when used alone, BCA has very weak antimicrobial activity. However, BCA in combination with CPFX shows potent antimicrobial effects against either FQ-resistant MRSA isolates or sensitive strains. These results were verified by the following two independent methods: Agar diffusion and time-kill curves. 
Table 2. In vitro interaction between $\mathrm{CPFX}$ and BCA.

\begin{tabular}{ccc}
\hline \multirow{2}{*}{ Strains } & \multicolumn{2}{c}{ Nonparametric method * } \\
\cline { 2 - 3 } & Fractional Inhibitory Concentration Index (FICI) \\
\cline { 2 - 3 } S. aureus 1662 & $0.25(0.25)$ & Interpretation \\
S. aureus 3633 & $0.16(0.06-0.16)$ & SYN \\
S. aureus 3814 & $0.38(0.25-0.50)$ & SYN \\
S. aureus 3725 & $0.19(0.09-0.31)$ & SYN \\
S. aureus 2855 & $0.13(0.06-0.25)$ & SYN \\
S. aureus 1881 & $0.38(0.38-0.50)$ & SYN \\
S. aureus 3014 & $0.38(0.25-0.5)$ & SYN \\
S. aureus 3115 & $0.50(0.38-0.50)$ & SYN \\
S. aureus 3451 & $0.16(0.08-0.31)$ & SYN \\
S. aureus 3182 & $0.38(0.38)$ & SYN \\
S. aureus 1787 & $0.25(0.25-0.38)$ & SYN \\
S.aureus ATCC 25923 & $0.50(0.31-0.50)$ & SYN \\
\hline
\end{tabular}

* SYN, synergism. For the FICI model, synergy was defined as a FICI of $\leq 0.5$, antagonism was defined as a FICI of $>4.0$, and indifference was defined as a FICI of $>0.5$ to 4 (i.e., no interaction).

\subsection{Time-Kill Curves}

Synergy with the drug combination was also seen in the time-kill curves (Figure 2). The time-kill studies were conducted using BCA and CPFX alone and in combination against one chosen clinical FQ-resistant MRSA 1662 isolate. After $24 \mathrm{~h}$, BCA $(32 \mu \mathrm{g} / \mathrm{mL})$ or CPFX $(32 \mu \mathrm{g} / \mathrm{mL})$ alone did not significantly affect the growth curve. The combination of CPFX and BCA had a higher antimicrobial effect than either drug alone. Given the initial inoculum of $10^{6} \mathrm{CFU} / \mathrm{mL}, \mathrm{CPFX}$ in combination with BCA resulted in a $2.6-\log _{10} \mathrm{CFU} / \mathrm{mL}$ decrease compared to $32 \mu \mathrm{g} / \mathrm{mL}$ CPFX alone at $24 \mathrm{~h}$ for isolate FQ-resistant MRSA 1662.

Figure 2. Time-kill curves of BCA and CPFX alone and in combination against clinical Methicillin -resistant $S$. aureus isolate 1662 . The strain at starting inocula of $10^{6} \mathrm{CFU} / \mathrm{mL}$ were exposed to in vivo-achievable concentrations of $32 \mu \mathrm{g} / \mathrm{mL}$ BCA alone and in combination with $64 \mu \mathrm{g} / \mathrm{mL}$ CPFX. At $0,3,6,24$, and $48 \mathrm{~h}$, aliquots were removed from each test tube to examine the cell metabolism ability. Symbols: $\bullet$, no drug control; 口, CPFX; $\boldsymbol{\Delta}, \mathrm{BCA} ; *$, CPFX plus BCA.

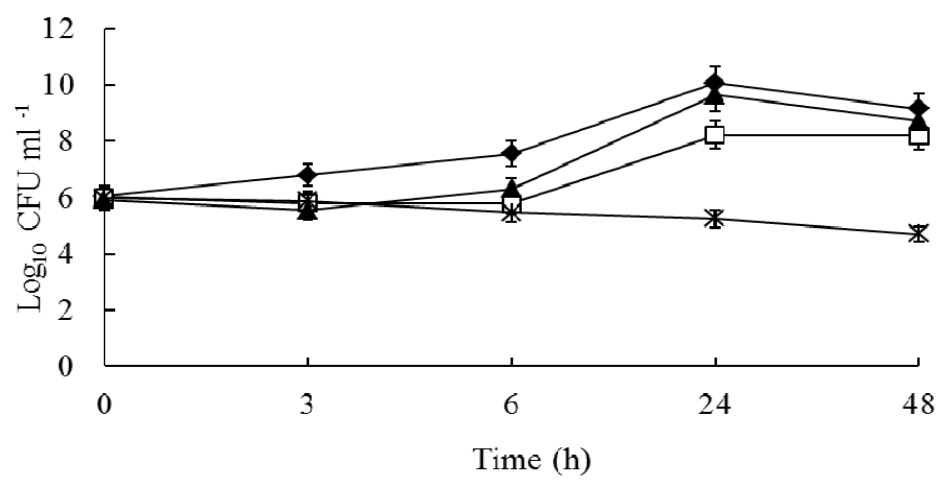




\subsection{Agar Diffusion Test}

The agar diffusion test was used to visually detect synergy between BCA and CPFX when combined (Figure 3). BCA alone $(64 \mu \mathrm{g})$ had no antimicrobial activity against the FQ-resistant MRSA 1662 isolate, while CPFX $(128 \mu \mathrm{g})$ showed weak antimicrobial activity. However, BCA (128 $\mu \mathrm{g}$ and $256 \mu \mathrm{g})$ in combination with CPFX $(128 \mu \mathrm{g})$ showed better antimicrobial effects. The combination of BCA and CPFX resulted in clearer and larger zones compared to either drug alone. The inhibition zone radius was increased to $10.0,13.5,14.0,15.5$ and $16.0 \mathrm{~mm}$ around the disks that contained CPFX $(128 \mu \mathrm{g})$ plus various amounts of $\mathrm{BCA}(16,32,64,128$, and $256 \mu \mathrm{g}$, respectively). These results were similar to the results of the checkerboard microdilution assay.

Figure 3. Agar disk diffusion assay with various concentrations of BCA alone (A) or in combination with $\mathrm{CPFX}$ (B) against $S$. aureus isolate MRSA 1662. Lables: A1, CONTROL; A2, BCA $32 \mu \mathrm{g} / \mathrm{mL}$; A3, BCA $64 \mu \mathrm{g} / \mathrm{mL}$; A4, BCA $128 \mu \mathrm{g} / \mathrm{mL}$; A5, BCA $256 \mu \mathrm{g} / \mathrm{mL}$; B1, CPFX $128 \mu \mathrm{g} / \mathrm{mL}$; B2, CPFX $128 \mu \mathrm{g} / \mathrm{mL}+$ BCA $16 \mu \mathrm{g} / \mathrm{mL}$; B3, CPFX $128 \mu \mathrm{g} / \mathrm{mL}+$ BCA $32 \mu \mathrm{g} / \mathrm{mL}$; B4, CPFX $128 \mu \mathrm{g} / \mathrm{mL}+$ BCA $64 \mu \mathrm{g} / \mathrm{mL} ;$ B5, CPFX $128 \mu \mathrm{g} / \mathrm{mL}+$ BCA $128 \mu \mathrm{g} / \mathrm{mL} ; \mathrm{B} 6$, CPFX $128 \mu \mathrm{g} / \mathrm{mL}+$ BCA $256 \mu \mathrm{g} / \mathrm{mL}$.

(A)

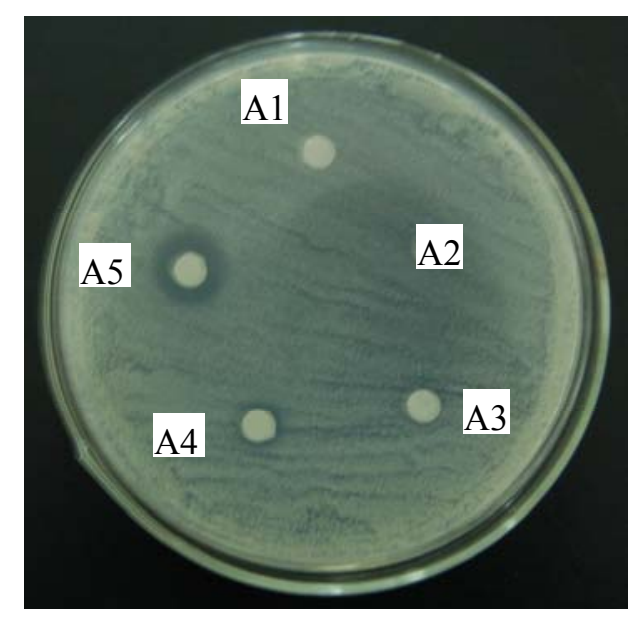

(B)

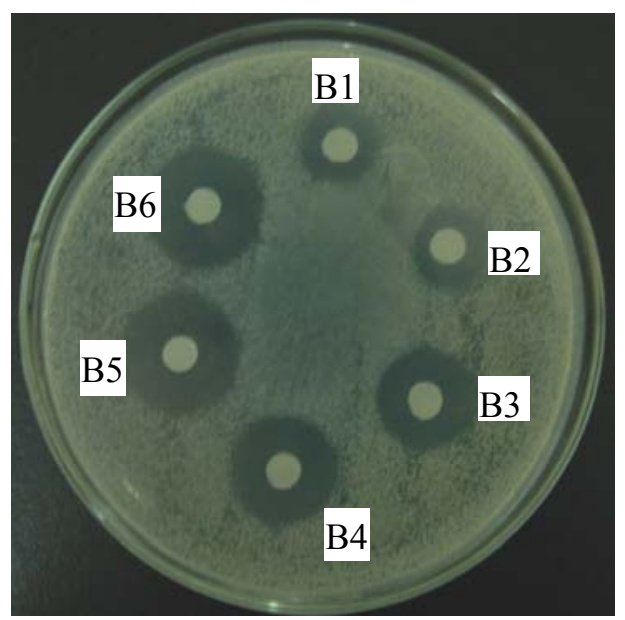

\section{Discussion}

In this study, we used the checkerboard microdilution method to analyze the effects of CPFX and BCA combinations on $S$. aureus. Subsequently, the time-kill and agar diffusion assays were used to further examine the nature of these interactions and to further analyze the interaction between BCA and CPFX against isolate FQ-resistant MRSA 1662 isolate.

We used the FICI to interpret our checkerboard method results, although interpretation of synergy or antagonism between drugs with the FICI method can be problematic. Because MIC determinations ares sensitive to dilution errors, the MIC is usually taken to be accurate to within one dilution when two-fold serial dilutions are used. Because the FICIs are determined from two drugs, the FICI value may differ between experiments. Therefore, in the interpretation of a single FICI, a value of $>4$ is considered to indicate antagonism, while a value of $\leq 0.5$ is considered to indicate synergy [17]. 
Previous studies have shown that when experiments were performed in triplicate for each strain combination studied, the results of the FICI model for all replicates could be interpreted as a single outcome (synergy, antagonism or indifference), thereby constraining the inter-experimental error. Thus, when the results of all three replicates were concordant, synergy or antagonism was claimed if FICIs were below 1 or above 1, respectively [18]. In the study, all cases show synergism.

The time-kill tubes were vortexed, a sample was removed at various time points, and the colony count was determined. Figure 2 clearly showed that the growth of FQ-resistant MRSA 1662 isolate exposed to the combination of CPFX and BCA was significantly inhibited compaierd to either drug alone, but the cells present in the inoculum were still viable upon transfer. This meant the effect was bacteriostatic, which is consistent with ciprofloxacin's mechanism of action. A bactericidal effect was defined as a $\geq 3-\log 10 \mathrm{CFU} / \mathrm{mL}$ decrease after $24 \mathrm{~h}$ of incubation compared to the size of the initial inoculum. Synergism was defined as a decrease in the colony count of $\geq 2-\log 10 \mathrm{CFU} / \mathrm{mL}$ with the combination compared to the count obtained with the most active single drug [31]. As the results showed, there was a 2.6- $\log _{10} \mathrm{CFU} / \mathrm{mL}$ decrease with CPFX in combination with BCA compared to $32 \mu \mathrm{g} / \mathrm{mL}$ CPFX alone at $24 \mathrm{~h}$ for isolate FQ-resistant MRSA 1662, which meant CPFX and BCA had synergetic bacteriostatic effect. For these experiments, the FICI analysis agreed well with the time-kill curves for the strains that were tested. The agar diffusion method can provide visual results of the interaction between BCA and CPFX. We found that the results of the agar diffusion, checkerboard microdilution and the time-kill experiments were in good agreement.

BCA is a selective drug that has strong antifungal activity [19-21] and can suppress the growth of various microorganisms [22]. The data from this study show that BCA alone has moderate MIC values for the methicillin-susceptible $S$. aureus strains. However, BCA can potentiate the antimicrobial activity of representative FQs (ciprofloxacin) against methicillin-resistant $S$. aureus strains, besides its direct inhibitory effect on microorganisms. The BCA/CPFX combination produced a powerful drug that was more effective than either drug alone. Flavonoids can complex with extracellular and soluble proteins and can thus complex with bacterial cell walls and disrupt microbial membranes $[8,23]$. Genistein is a topoisomerase inhibitor that has a structure similar to BCA (except for one surplus methyl group at the 4' position); thus, BCA may also inhibit DNA topoisomerases, which participate in various aspects of DNA metabolism to inhibit microorganisms, the interference related metabolic pathways may be responsible for the antimicrobial action of BCA [12]. A study by Lee showed that a methoxyl group and a hydroxyl group at the 5' position were essential for antimicrobial activity [12], and both of these criteria are present in the BCA structure (Figure 1).

FQs are some of the most frequently prescribed antimicrobial agents worldwide. FQs interact with DNA gyrase, bacterial type II topoisomerase gyrase, and topoisomerase IV, which leads to changes in DNA supercoiling and ultimately affects gene transcription and DNA metabolism [24-26]. Previous study showed FQs can induce the SOS response by comparison of a wild-type $S$. aureus strain, which is a critical response to environmental stress [27]. Thus, FQs increase the longevity of the normally short-lived DNA-topoisomerase cleavage intermediates [28]. These intermediates interfere with the DNA machinery, which results in multiple deleterious effects, such as chromosome fragmentation, inhibition of DNA synthesis, and death [29]. Thus, BCA and CPFX have their own antimicrobial mechanism, it is necessary to determine the underlying mechanism of BCA/CPFX synergy in our further study. 


\section{Experimental}

\subsection{Bacterial Strains and Reagents}

Eleven clinical MRSA isolates were obtained from blood samples of infected patients who were admitted to the First Hospital of Jilin University during the year 2008-2009. The quality control (QC) strain ATCC 25923 was obtained from the China Medical Culture Collection Center (CMCC). BCA, dimethyl sulfoxide (DMSO) and CPFX were purchased from Sigma (St. Louis, MO, USA).

\subsection{Medium}

Mueller-Hinton broth (MHB) and agar (MHA) were purchased from BD Biosciences, Inc. (Sparks, MD, USA). The $S$. aureus colony counts were determined from growth on the MHA.

\subsection{Drug Susceptibility Testing}

The MICs of drugs were determined by microdilution of the drug in MHB, which was performed as recommended by the Clinical and Laboratory Standards Institute (CLSI, formerly NCCLS [30]). The strains were plated on MHA and incubated at $37{ }^{\circ} \mathrm{C}$ overnight. Colonies from the plates were resuspended in $\mathrm{MHB}$, and the concentration was adjusted to an $\mathrm{OD}_{660}$ of $0.1\left(\sim 10^{8} \mathrm{CFU} / \mathrm{mL}\right.$, which matches a $0.5 \mathrm{McF}$ arland turbidity standard). The inoculum was diluted in MHB to a concentration of $10^{6} \mathrm{CFU} / \mathrm{mL}$. Serial two-fold dilutions of the antimicrobial agents were prepared in MHB to obtain the required range of concentrations. The assay was performed in 96-well microtiter plates, and a positive control, which contained inoculated broth without drugs, was included in every plate. Fifty microliters of the diluted antimicrobial drug was plated into individual wells in triplicate. Fifty microliters of the bacterial inoculum was added to each well, and the plate was incubated at $37{ }^{\circ} \mathrm{C}$ for $24 \mathrm{~h}$. Bacterial growth was examined by measuring the optical density at $660 \mathrm{~nm}$ with a microplate spectrophotometer. The MIC was defined as the lowest concentration of drug that inhibited $>90 \%$ of the microorganism's growth [17]. Each isolate was tested in triplicate on different days.

\subsection{Checkerboard Assay}

Interactions between CPFX and BCA were assessed with a microbroth checkerboard technique. Serial two-fold dilutions of BCA and CPFX were prepared in MHB that ranged from 1/512 to 4-fold MIC concentration. The checkerboard plates were inoculated with $10^{6} \mathrm{CFU} / \mathrm{mL}$ of MRSA 1662 and incubated for $24 \mathrm{~h}$ at $37{ }^{\circ} \mathrm{C}$. The growth in each well was quantified spectrophotometrically as previously described above, and the background $\mathrm{OD}_{660}$ was subtracted from the $\mathrm{OD}_{660}$ of each well. The percentage of growth in each well was calculated by dividing the $\mathrm{OD}_{660}$ of each well by the $\mathrm{OD}_{660}$ of the drug-free well, and the plates were handled according to the protocol previously published by Li et al. [18]. Each isolate was tested in triplicate on different days.

\subsection{Drug Interaction Interpretation}

To study the interaction of BCA in combination with CPFX against $S$. aureus, the data were analyzed with the models: The fractional inhibitory concentration index (FICI). 
The FICI was calculated as follows: $\mathrm{FICI}=\mathrm{FIC}_{\mathrm{A}}+\mathrm{FIC}_{\mathrm{B}}$, where $\mathrm{FIC}_{\mathrm{A}}=\mathrm{MIC}$ of drug $\mathrm{A}$ in the combination divided by the MIC of drug $\mathrm{A}$ alone, and $\mathrm{FIC}_{\mathrm{B}}=\mathrm{MIC}$ of drug $\mathrm{B}$ in the combination divided by the MIC of drug B alone. Synergy was defined when the FICI was $\leq 0.5$, indifference when the FICI fell in the range of 0.5-4, and antagonism was defined as an FICI $>4$ [17].

\subsection{Time-Kill Curves}

The tested strain MRSA 1662 was prepared at a concentration of $10^{6} \mathrm{CFU} / \mathrm{mL}$ in MHB [31]. Tubes of broth were prepared containing CPFX alone at a concentration of $1 / 8$ the MIC, BCA alone at a concentration of 1/8 the MIC, and a combination of both drugs (1/8 the MIC of CPFX and 1/8 the MIC of BCA). A control tube without drugs was included in each series. At predetermined time points $(0,3$, 6,24 , and $48 \mathrm{~h}$ ), a $100-\mu \mathrm{L}$ sample was removed from each test suspension, serially diluted in MHB, and plated on MHA plates to determine the colony count [32]. The experiment was performed triplicately.

A bactericidal effect was defined as a $\geq 3-\log 10 \mathrm{CFU} / \mathrm{mL}$ decrease after $24 \mathrm{~h}$ of incubation compared to the size of the initial inoculum. Synergism was defined as a decrease in the colony count of $\geq 2-\log 10 \mathrm{CFU} / \mathrm{mL}$ with the combination compared to the count obtained with the most active single drug. Antagonism was defined as an increase in the colony count of $\geq 2-\log 10 \mathrm{CFU} / \mathrm{mL}$ with the combination compared to the count obtained with the most active single drug [31].

\subsection{Agar Diffusion Test}

The interaction between BCA and CPFX was tested with an agar diffusion assay. Disks containing BCA and CPFX alone and in combination were placed on the MHA plates spread with MRSA 1662 [33]. After incubation for $24 \mathrm{~h}$ at $37^{\circ} \mathrm{C}$, the zone of inhibition was measured.

\section{Conclusions}

In summary, this is first report that BCA displays synergistic activity in combination with the antibacterial agent CPFX against $S$. aureus, which might provide an alternative approach to overcome antimicrobial drug resistance.

\section{Acknowledgements}

This work was supported by the National Nature Science Foundation of China (Number 30871889), the State Key Laboratory for Molecular Virology and Genetic Engineering (Number 2011KF05), the Specialized Research Fund for the Doctoral Program of Higher Education (SRFDP) (Number 200801831051), the Fund for Science and Technology Development of the Jilin Province, China (Number 200705233), and the Fundamental Research Funds for the Central Universities.

\section{Conflict of interest}

The authors declare that they have no conflict of interest. 


\section{Reference and Notes}

1. Li, D.; Cheung, A. Repression of hla by rot is dependent on sae in Staphylococcus aureus. Infect. Immun. 2008, 76, 1068-1075.

2. Klevens, R.M.; Gorwitz, R.J.; Collins, A.S. Methicillin-resistant Staphylococcus aureus: A primer for dentists. J. Am. Dent. Assoc. 2008, 139, 1328-1337.

3. Ayliffe, G.A. The progressive intercontinental spread of methicillin-resistant Staphylococcus aureus. Clin. Infect. Dis. 1997, 24 (Suppl. 1), S74-S79.

4. Paulsen, I.T.; Firth, M.; Skurray, R.A. Resistance to antimicrobial agents other than b-lactams. In The Staphylococci in Human Disease; Crossley, K.B., Archer, G.L., Eds.; Churchill Livingstone: New York, NY, USA, 1997; pp. 175-212.

5. Kuroda, H.; Kuroda, M.; Cui, L.; Hiramatsu, K. Subinhibitory concentrations of beta-lactam induce haemolytic activity in Staphylococcus aureus through the SaeRS two-component system. FEMS Microbiol. Lett. 2007, 268, 98-105.

6. Rios, J.L.; Recio, M.C. Medicinal plants and antimicrobial activity. J. Ethnopharmacol. 2005, 100, 80-84.

7. Li, L.; Li, Z.; Guo, N.; Jin, J.; Du, R.; Liang, J.; Wu, X.; Wang, X.; Liu, M.; Jin, Q.; Yu, L. Synergistic activity of 1-(1-naphthylmethyl)-piperazine with ciprofloxacin against clinically resistant Staphylococcus aureus, as determined by different methods. Lett. Appl. Microbiol. 2011, 52, 372-378.

8. Cushnie, T.P.T.; Lamb, A.J. Antimicrobial activity of flavonoids. Int. J. Antimicrob. Agents 2005, 26, 343-356.

9. Lechner, D.; Gibbons, S.; Bucar, F. Plant phenolic compounds as ethidium bromide efflux inhibitors in Mycobacterium smegmatis. J. Antimicrob. Chemother. 2008, 62, 345-348.

10. Dastidar, S.G.; Manna, A.; Kumar, K.A.; Mazumdar, K.; Dutta, N.K.; Chakrabarty, A.N.; Motohashi, N.; Shirataki, Y. Studies on the antibacterial potentiality of isoflavones. Int. J. Antimicrob. Agents 2004, 23, 99-102.

11. Flythe, M.; Kagan, I. Antimicrobial effect of red clover (Trifolium pratense) phenolic extract on the ruminal hyper ammonia-producing bacterium, Clostridium sticklandii. Curr. Microbiol. 2010, 61, 125-131.

12. Sklenickova, O.; Flesar, J.; Kokoska, L.; Vlkova, E.; Halamova, K.; Malik, J. Selective growth inhibitory effect of biochanin A against intestinal tract colonizing bacteria. Molecules 2010, 15, 1270-1279.

13. Medjakovic, S.; Jungbauer, A. Red clover isoflavones biochanin A and formononetin are potent ligands of the human aryl hydrocarbon receptor. J. Steroid. Biochem. Mol. Biol. 2008, 108, 171-177.

14. Shen, P.; Liu, M.H.; Ng, T.Y.; Chan, Y.H.; Yong, E.L. Differential effects of isoflavones, from Astragalus membranaceus and Pueraria thomsonii, on the activation of PPARa, PPARg, and adipocyte differentiation in vitro. J. Nutr. 2006, 136, 899-905.

15. Lee, Y.S.; Seo, J.S.; Chung, H.T.; Jang, J.J. Inhibitory effects of biochanin A on mouse lung tumor induced by benzo(a)pyrene. J. Kor. Med. Sci. 1991, 6, 325-328. 
16. Cassady, J.M.; Zennie, T.M.; Chae, Y.H.; Ferin, M.A.; Portuondo, N.E.; Baird, W.M. Use of a mammalian cell culture benzo(a)pyrene metabolism assay for the detection of potential anticarcinogens from natural products: Inhibition of metabolism by biochanin A, an isoflavone from Trifolium pratense L. Cancer Res. 1988, 48, 6257-6261.

17. Oo, T.Z.; Cole, N.; Garthwaite, L.; Willcox, M.D.; Zhu, H. Evaluation of synergistic activity of bovine lactoferricin with antibiotics in corneal infection. J. Antimicrob. Chemother. 2010, 65, 1243-1251.

18. Li, Y.; Sun, S.; Guo, Q.; Ma, L.; Shi, C.; Su, L.; Li, H. In vitro interaction between azoles and cyclosporin a against clinical isolates of Candida albicans determined by the chequerboard method and time-kill curves. J. Antimicrob. Chemother. 2008, 61, 577-585.

19. Weidenborner, M.; Hindorf, H.; Jha, H.C.; Tsotsonos, P.; Egge, H. Antifungal activity of isoflavonoids in different reduced stages on Rhizoctonia solani and Sclerotium rolfsii. Phytochemistry 1990, 29, 801-803.

20. Kramer, R.P.; Hindorf, H.; Jha, H.C.; Kallage, J.; Zilliken, F. Antifungal activity of soybean and chickpea isoflavonec and their reduced derivatives. Phytochemistry 1984, 23, 2203-2205.

21. Rojas, R.; Bustamante, B.; Ventosilla, P.; Fernadez, I.; Caviedes, L.; Gilman, R.H.; Lock, O.; Hammond, G.B. Larvicidal, antimycobacterial and antifungal compounds from the bark of the Peruvian plant Swartzia polyphylla DC. Chem. Pharm. Bull. 2006, 54, 278-279.

22. Sklenickova, O.; Flesar, J.; Kokoska, L. Selective antimicrobial activity of isoflavonoids. Ann. Nutr. Metab. 2009, 55, 391.

23. Tsuchiya, H.; Sato, M.; Miyazaki, T.; Fujiwara, S.; Tanigaki, S.; Ohyama, M.; Tanaka, T.; Iinuma, M. Comparative study on the antibacterial activity of phytochemical flavanones against methicillin-resistant Staphylococcus aureus. J. Ethnopharmacol. 1996, 50, $27-34$.

24. Drlica, K.; Zhao, X. DNA gyrase, topoisomerase IV, and the 4-quinolones. Microbiol. Mol. Biol. Rev. 1997, 61, 377-392.

25. Hooper, D.C. Mechanisms of fluoroquinolone resistance. Drug Resist. Updat. 1999, 2, 38-55.

26. Becnel Boyd, L.; Maynard, M.J.; Morgan-Linnell, S.K.; Horton, L.B.; Sucgang, R.; Hamil, R.J.; Jimenez, J.R.; Versalovic, J.; Steffen, D.; Zechiedrich, L. Relationships among ciprofloxacin, gatifloxacin, levofloxacin, and norfloxacin MICs for fluoroquinolone-resistant Escherichia coli Clinical Isolates. Antimicrob. Agents Chemother. 2009, 53, 229-234.

27. Cirz, R.T.; Jones, M.B.; Gingles, N.A.; Minogue, T.D.; Jarrahi, B.; Peterson, S.N.; Romesberg, F.E. Complete and SOS-mediated response of Staphylococcus aureus to the antibiotic ciprofloxacin. J. Bacteriol. 2007, 189, 531-539.

28. Froelich-Ammon, S.J.; Osheroff, N. Topoisomerase poisons: Harnessing the dark side of enzyme mechanism. J. Biol. Chem. 1995, 270, 21429-21432.

29. Drlica, K.; Malik, M.; Kerns, R.J.; Zhao, X. Quinolone-mediated bacterial death. Antimicrob. Agents Chemother. 2008, 52, 385-392.

30. Clinical and Laboratory Standards Institute. Methods for Dilution Antimicrobial Susceptibility Tests for Bacteria That Grow Aerobically, 8th ed.; CLSI: Wayne, PA, USA, 2009. 
31. Jacqueline, C.; Navas, D.; Batard, E.; Miegeville, A.F.; Le Mabecque, V.; Kergueris, M.F.; Bugnon, D.; Potel, G.; Caillon, J. In vitro and in vivo synergistic activities of linezolid combined with subinhibitory concentrations of imipenem against methicillin-resistant Staphylococcus aureus. Antimicrob. Agents Chemother. 2005, 49, 45-51.

32. Santos, A.O.; Ueda-Nakamura, T.; Dias Filho, B.P.; Veiga Junior, V.F.; Pinto, A.C.; Nakamura, C.V. Antimicrobial activity of Brazilian copaiba oils obtained from different species of the Copaifera genus. Mem. Inst. Oswaldo Cruz 2008, 103, 277-281.

33. Périchon, B.; Courvalin, P. Synergism between $\beta$-lactams and glycopeptides against VanA-type methicillin-resistant Staphylococcus aureus and heterologous expression of the vanA operon. Antimicrob. Agents Chemother. 2006, 50, 3622-3630.

Sample Availability: Samples of the compounds biochanin A is available from the authors.

(C) 2011 by the authors; licensee MDPI, Basel, Switzerland. This article is an open access article distributed under the terms and conditions of the Creative Commons Attribution license (http://creativecommons.org/licenses/by/3.0/). 